\title{
ON OPTIMAL STOPPING PROBLEMS FOR MATRIX-EXPONENTIAL JUMP-DIFFUSION PROCESSES
}

\author{
YUAN-CHUNG SHEU ${ }^{* * *}$ AND \\ MING-YAO TSAI,* National Chiao Tung University
}

\begin{abstract}
In this paper we consider optimal stopping problems for a general class of reward functions under matrix-exponential jump-diffusion processes. Given an American call-type reward function in this class, following the averaging problem approach (see, for example, Alili and Kyprianou (2005), Kyprianou and Surya (2005), Novikov and Shiryaev (2007), and Surya (2007)), we give an explicit formula for solutions of the corresponding averaging problem. Based on this explicit formula, we obtain the optimal level and the value function for American call-type optimal stopping problems.
\end{abstract}

Keywords: Optimal stopping problem; American call-type reward function; averaging problem; matrix-exponential distribution; jump-diffusion process

2010 Mathematics Subject Classification: Primary 60G40; 60J75; 60G51

\section{Introduction}

The optimal stopping problems we consider in this paper will be of the form

$$
V(x)=\sup _{\tau \in \mathcal{T}} \mathrm{E}_{x}\left(\mathrm{e}^{-r \tau} g\left(X_{\tau}\right)\right)
$$

where $X=\left\{X_{t}: t \geq 0\right\}$ under $\mathrm{P}_{x}$ is a Lévy process started from $X_{0}=x, g$ is a continuous reward function, $r>0$, and $\mathcal{T}$ is a family of stopping times with respect to the natural filtration generated by $X$. The optimal stopping problem consists of finding the value function $V(x)$ and the optimal stopping time $\tau^{*}$ such that $V(x)=\mathrm{E}_{x}\left(\mathrm{e}^{-r \tau^{*}} g\left(X_{\tau^{*}}\right)\right)$. The general theory of optimal stopping rules for Markov processes says that the value function $V(x)$ is the smallest $r$-excessive majorant of $g(x)$. Moreover, the first entry time $\tau_{D}$ of the process $X$ into the stopping region $D=\{x \in \mathbb{R}, V(x)=g(x)\}$ is the smallest optimal stopping time (if it exists) and, hence, the value function is given by the formula $\mathrm{E}_{x}\left(\mathrm{e}^{-r \tau_{D}} g\left(X_{\tau_{D}}\right)\right)$.

For diffusion processes, many authors have determined the boundary of the stopping region $D$ using the smooth pasting condition for the value function and solved the optimal stopping problem by reducing it to the problem of solving a corresponding Stefan free boundary problem. Unfortunately, once problem (1.1) is driven by Lévy processes, the smooth pasting condition may break down. (See [1] for an example.) Recently, to solve the optimal stopping problem (1.1) in a general setting, Surya [13] proposed using an averaging problem approach instead of one of the many other methods given in the literature. (For earlier works on the averaging problem

Received 12 April 2011; revision received 18 November 2011.

* Postal address: Department of Applied Mathematics, National Chiao Tung University, Hsinchu, Taiwan.

** Email address: sheu@math.nctu.edu.tw

Partially supported by NSC grant NSC100-2115-M-009-006, CMMSC, and NCTS, Taiwan. 
approach, see [1], [4], [8], and [12].) The averaging problem approach does not appeal to a free boundary problem associated to the optimal stopping problem. Instead, by solving the corresponding averaging problem, we have a new fluctuation identity for overshoots of a Lévy process. With this identity, Surya [13] showed by martingale arguments that an optimal solution can be found if the solutions to the averaging problem have certain monotonicity properties. By this approach, he was able to reproduce the results of, among others, Kyprianou and Surya [8], Mordecki [10], and Novikov and Shiryaev [12].

In this paper we consider a matrix-exponential jump-diffusion process $X$ of the form given in (2.2) below. For a general class of reward functions, under some assumptions on the process, we give an explicit formula in Theorem 3.1 for solutions of the averaging problem. (Our result depends on the recent work of Lewis and Mordecki [9].) Moreover, when the reward function $g$ is of American call type, we derive sufficient criteria for optimality in terms of the reward function $g$ and the solutions of the averaging problem (see Theorem 3.2). The class of reward functions contains many known examples in the literature and our results are consistent with those of Kyprianou and Surya [8], Mordecki [10], Boyarchenko and Levendorskii [4], and many others. Also, our examples in Section 4 show that the sufficient conditions for optimality in Theorem 3.2 are easier to verify than those given in the literature.

The rest of the paper is organized as follows. In Section 2 we recall some main results of Surya [13] and Lewis and Mordecki [9]. In Section 3 we present our main results. In Section 4 we give some examples.

\section{Preliminaries}

\subsection{Sufficient conditions for optimality}

Let $X=\left\{X_{t}: t \geq 0\right\}$ be a real-valued Lévy process defined on $\left(\Omega, \mathcal{F},\left\{\mathcal{F}_{t}\right\}, \mathrm{P}\right)$ such that $X_{0}=0$ almost surely. A Lévy process starting from $X_{0}=x$ is simply defined as $x+X_{t}$ for $t \geq 0$, and we denote its law by $\mathrm{P}_{x}$. For every Lévy process, we have $\mathrm{E}\left(\mathrm{e}^{\mathrm{i} u X_{t}}\right)=\mathrm{e}^{t \psi(u)}$, where $\psi$ is called the characteristic exponent of $X$ and is given by the formula

$$
\psi(u)=\mathrm{i} a u-\frac{1}{2} b^{2} u^{2}+\int_{\mathbb{R}}\left(\mathrm{e}^{\mathrm{i} u x}-1-\mathrm{i} u x \mathbf{1}_{\{|x|<1\}}\right) \pi(\mathrm{d} x) .
$$

Here $a \in \mathbb{R}, b \geq 0$, and $\pi$ is a measure on $\mathbb{R} \backslash\{0\}$ such that $\int_{\mathbb{R}}\left(1 \wedge x^{2}\right) \pi(\mathrm{d} x)<\infty$.

Denote by $e_{r}$ an exponential random variable with parameter $r>0$, independent of the process $X$, and denote by $M_{r}=\sup _{0 \leq s \leq e_{r}} X_{s}$ and $I_{r}=\inf _{0 \leq s \leq e_{r}} X_{s}$ the supremum and the infimum of the Lévy process $X$ killed at the independent exponential random time $e_{r}$. It is well known that $X_{e_{r}}-I_{r}$ and $I_{r}$ are independent, and that $X_{e_{r}}-I_{r}$ has the same distribution as $M_{r}$. From these, we obtain the Wiener-Hopf factorization formula: $\mathrm{Ee}^{\mathrm{i} u X_{e_{r}}}=r /(r-\psi(u))=$ $\psi_{r}^{+}(u) \psi_{r}^{-}(u)$, where $\psi_{r}^{+}(u)=\mathrm{E}\left(\mathrm{e}^{\mathrm{i} u M_{r}}\right)$ and $\psi_{r}^{-}(u)=\mathrm{E}\left(\mathrm{e}^{\mathrm{i} u I_{r}}\right)$.

To solve the optimal stopping problem (1.1), as in [8], [12], [13], and many other works, we first introduce the corresponding averaging problem. We say that the reward function $g$ is of American call type if $g$ is a nondecreasing continuous function and $\{g>0\}=(\hat{a}, \infty)$ for some $\hat{a}<\infty$. Given a reward function $g$ of American call type and $r>0$, the averaging problem for the optimal stopping problem (1.1) consists of finding a function $\tilde{Q}_{g}$ satisfying the condition

$$
\mathrm{E}\left(\tilde{Q}_{g}\left(x+M_{r}\right)\right)=g(x)
$$

for every $x>\hat{a}$. Using similar arguments (with some minor modifications) as in [13], we have the following sufficient conditions for optimality. 
Theorem 2.1. Given a reward function $g$ of American call type with $H=\{g>0\}=(\hat{a}, \infty)$ for some $\hat{a}<\infty$, consider a continuous function $\tilde{Q}_{g}$ on $H$ satisfying the averaging condition (2.1) for every $x \in H$. We assume further that there exists $\hat{x} \in H$ such that $\tilde{Q}_{g}(\hat{x})=0$, $\tilde{Q}_{g}(x)$ is nondecreasing for $x>\hat{x}$ and $\tilde{Q}_{g}(x) \leq 0$ for $\hat{a}<x<\hat{x}$. Let $x^{*}$ be the largest root of $\tilde{Q}_{g}(x)=0$ in $(\hat{a}, \infty)$, and set $\tau^{*}=\inf \left\{t>0: X_{t}>x^{*}\right\}$. Then $\tau^{*}$ is the optimal stopping time for the optimal stopping problem (1.1) and the value function is given by $V(x)=$ $\mathrm{E}\left(\tilde{Q}_{g}\left(x+M_{r}\right) \mathbf{1}_{\left\{x+M_{r}>x^{*}\right\}}\right)$.

Remark 2.1. (a) By replacing $X$ with its dual process $\hat{X}=-X$ and $x$ with $-x$, similar results were also obtained in Surya [13] for American put-type optimal stopping problems.

(b) It is worth noting that if $g(x)=\mathrm{E}\left[\int_{0}^{\infty} \mathrm{e}^{-r t} h\left(x+X_{t}\right) \mathrm{d} t\right]$ for some bounded function $h$ then, by the Wiener-Hopf fluctuation identity, the function $\tilde{Q}_{g}(x)=\mathrm{E}_{x}\left[h\left(I_{r}\right)\right]$ is a solution of the averaging problem (2.1).

\subsection{Matrix-exponential jump-diffusion processes}

From now on, we consider matrix-exponential jump-diffusion processes $X$ of the form

$$
X_{t}=X_{0}+a t+b W_{t}+\sum_{n=1}^{N_{t}^{\lambda}} Y_{n}+\sum_{k=1}^{N_{t}^{\mu}} Z_{k}, \quad t \geq 0
$$

Here $a \in \mathbb{R} \backslash\{0\}, b \geq 0, W=\left(W_{t}, t \geq 0\right)$ is a standard Brownian motion, and $N^{\lambda}=\left(N_{t}^{\lambda}\right.$; $t \geq 0)$ and $N^{\mu}=\left(N_{t}^{\mu} ; t \geq 0\right)$ are the Poisson processes with rates $\lambda>0$ and $\mu>0$, respectively. Also, $Y=\left(Y_{n}, n \in \mathbb{N}\right)$ and $Z=\left(Z_{k}, k \in \mathbb{N}\right)$ are sequences of independent random variables with identical matrix-exponential distributions given by

$$
\mathrm{d} F^{(+)}(x)=\mathbf{1}_{\{x>0\}} \sum_{k=1}^{v_{1}} \sum_{j=1}^{n_{k}} \frac{c_{k j} \beta_{k}^{j} x^{j-1}}{(j-1) !} \mathrm{e}^{-\beta_{k} x} \mathrm{~d} x
$$

and

$$
\mathrm{d} F^{(-)}(x)=\mathbf{1}_{\{x<0\}} \sum_{p=1}^{v_{2}} \sum_{m=1}^{\ell_{p}} \frac{\tilde{c}_{p m} \alpha_{p}^{m}(-x)^{m-1}}{(m-1) !} \mathrm{e}^{\alpha_{p} x} \mathrm{~d} x,
$$

respectively. Here the parameters $c_{k j}, \beta_{k}, \tilde{c}_{p m}$, and $\alpha_{p}$ can in principle take complex values, but if we order $\alpha_{p}$ and $\beta_{k}$ by their real parts then $\alpha_{1}$ and $\beta_{1}$ must be real, while the others may be complex with $0<\beta_{1}<\operatorname{Re}\left(\beta_{2}\right) \leq \cdots \leq \operatorname{Re}\left(\beta_{v_{1}}\right)$ and $0<\alpha_{1}<\operatorname{Re}\left(\alpha_{2}\right) \leq \cdots \leq \operatorname{Re}\left(\alpha_{v_{2}}\right)$. (For more on matrix-exponential distributions, see, for example, [2].) The random variables $W, N^{\lambda}, N^{\mu}, Y$, and $Z$ are assumed to be independent. Note that the characteristic exponent of $X$ is given by

$$
\psi(z)=\mathrm{i} a z-\frac{b^{2} z^{2}}{2}+\lambda\left[\sum_{k=1}^{v_{1}} \sum_{j=1}^{n_{k}} c_{k j}\left(\frac{\mathrm{i} \beta_{k}}{z+\mathrm{i} \beta_{k}}\right)^{j}-1\right]+\mu\left[\sum_{p=1}^{v_{2}} \sum_{m=1}^{\ell_{p}} \tilde{c}_{p m}\left(\frac{-\mathrm{i} \alpha_{p}}{z-\mathrm{i} \alpha_{p}}\right)^{m}-1\right] .
$$

We quote the following results of Lewis and Mordecki [9].

Theorem 2.2. (a) The equation $r-\psi(z)=0$ has, in the half-plane $\operatorname{Im}(z)<0, \mu_{1}$ distinct roots $-\mathrm{i} \rho_{1}, \ldots,-\mathrm{i} \rho_{\mu_{1}}$ (with respective multiplicities $m_{1}, \ldots, m_{\mu_{1}}$ ), ordered such that $0<$ $\operatorname{Re}\left(\rho_{1}\right) \leq \operatorname{Re}\left(\rho_{2}\right) \leq \cdots \leq \operatorname{Re}\left(\rho_{\mu_{1}}\right)$. The root $-\mathrm{i} \rho_{1}$ is purely imaginary. Furthermore, the 
total root count $\sum_{j=1}^{\mu_{1}} m_{j}$ is equal to $\bar{n}$ when $-X^{-}$is a subordinator and $\bar{n}+1$ when $-X^{-}$is not a subordinator. Here, $\bar{n}=\sum_{k=1}^{v_{1}} n_{k}$. On the other hand, the equation $r-\psi(z)=0$ has, in the half-plane $\operatorname{Im}(z)>0, \mu_{2}$ distinct roots $-\mathrm{i} \tilde{\rho}_{1}, \ldots,-\mathrm{i} \tilde{\rho}_{\mu_{2}}$ (with respective multiplicities $\left.\tilde{m}_{1}, \ldots, \tilde{m}_{\mu_{2}}\right)$, ordered such that $\operatorname{Re}\left(\tilde{\rho}_{\mu_{2}}\right) \leq \cdots \leq \operatorname{Re}\left(\tilde{\rho}_{1}\right)<0$. The root $-\mathrm{i} \tilde{\rho}_{1}$ is purely imaginary. Furthermore, the total root count $\sum_{j=1}^{\mu_{2}} \tilde{m}_{j}$ is equal to $\bar{\ell}$ when $X^{+}$is a subordinator and $\bar{\ell}+1$ when $X^{+}$is not a subordinator. Here, $\bar{\ell}=\sum_{p=1}^{v_{2}} \ell_{p}$.

(b) The Laplace transform $\psi_{r}^{-}$for $I_{r}$ is given by

$$
\psi_{r}^{-}(u)=\prod_{k=1}^{v_{2}}\left(\frac{u-\mathrm{i} \alpha_{k}}{-\mathrm{i} \alpha_{k}}\right)^{\ell_{k}} \prod_{j=1}^{\mu_{2}}\left(\frac{\mathrm{i} \tilde{\rho}_{j}}{u+\mathrm{i} \tilde{\rho}_{j}}\right)^{\tilde{m}_{j}}
$$

and the Laplace transform $\psi_{r}^{+}$for $M_{r}$ is given by

$$
\psi_{r}^{+}(u)=\prod_{k=1}^{v_{1}}\left(\frac{u+\mathrm{i} \beta_{k}}{\mathrm{i} \beta_{k}}\right)^{n_{k}} \prod_{j=1}^{\mu_{1}}\left(\frac{\mathrm{i} \rho_{j}}{u+\mathrm{i} \rho_{j}}\right)^{m_{j}} .
$$

\section{Main results}

Recall that $\left\{X_{t}\right\}_{t \geq 0}$ is a matrix-exponential jump-diffusion process of the form given in (2.2) and $-\mathrm{i} \tilde{\rho}_{1}, \ldots,-\mathrm{i} \tilde{\rho}_{\mu_{2}},-\mathrm{i} \rho_{1}, \ldots,-\mathrm{i} \rho_{\mu_{1}}$ are distinct roots of $r-\psi(z)=0$ with $\operatorname{Re}\left(\tilde{\rho}_{\mu_{2}}\right) \leq$ $\cdots \leq \operatorname{Re}\left(\tilde{\rho}_{1}\right) \leq 0<\operatorname{Re}\left(\rho_{1}\right) \leq \cdots \leq \operatorname{Re}\left(\rho_{\mu_{1}}\right)$. We assume further that all the roots are simple, i.e. $m_{1}=\cdots=m_{\mu_{1}}=\tilde{m}_{1}=\cdots=\tilde{m}_{\mu_{2}}=1$. (This is the case for hyperexponential jump-diffusion models. Owing to its analytical tractability, this model has gained popularity among practitioners and academics working in mathematical finance and insurance. In fact, in the literature, there are many related works which also cover the case of multiple roots. See, for example, [3], [5], and [7].) Then, under these assumptions, we solve the optimal stopping problem (1.1) for a class of continuous reward functions $g$ and $r>0$.

First we observe, by Theorem 2.2, that the distribution of $\inf _{0 \leq s \leq e_{r}} X_{s}$ is given by

$$
\mathrm{P}\left(\inf _{0 \leq s \leq e_{r}} X_{s} \in \mathrm{d} y\right)=\mathbf{1}_{\{a>0, b=0\}} \tilde{d}_{0} \delta_{0}(\mathrm{~d} y)+\mathbf{1}_{\left\{\mu_{2} \geq 1\right\}}\left[\left(\sum_{\eta=1}^{\mu_{2}} \tilde{d}_{\eta} \tilde{\rho}_{\eta} \mathrm{e}^{-\tilde{\rho}_{\eta} y}\right) \mathbf{1}_{\{y<0\}} \mathrm{d} y\right],
$$

where $\tilde{d}_{0}=\prod_{j=1}^{\mu_{2}}\left(-\tilde{\rho}_{j}\right) \prod_{k=1}^{v_{2}} \alpha_{k}^{-\ell_{k}}$ and

$$
\tilde{d}_{j}=-\prod_{k=1}^{v_{2}}\left(\frac{\tilde{\rho}_{j}+\alpha_{k}}{\alpha_{k}}\right)^{\ell_{k}} \prod_{m=1, m \neq j}^{\mu_{2}} \frac{\tilde{\rho}_{m}}{-\tilde{\rho}_{j}+\tilde{\rho}_{m}} \quad \text { for } 1 \leq j \leq \mu_{2} .
$$

Also, the distribution of $\sup _{0 \leq s \leq e_{r}} X_{s}$ is given by

$$
\mathrm{P}\left(\sup _{0 \leq s \leq e_{r}} X_{s} \in \mathrm{d} y\right)=\mathbf{1}_{\{a<0, b=0\}} d_{0} \delta_{0}(\mathrm{~d} y)+\mathbf{1}_{\left\{\mu_{1} \geq 1\right\}}\left[\left(\sum_{j=1}^{\mu_{1}} d_{j} \rho_{j} \mathrm{e}^{-\rho_{j} y}\right) \mathbf{1}_{\{y>0\}} \mathrm{d} y\right],
$$

where $d_{0}=\prod_{j=1}^{\mu_{1}} \rho_{j} \prod_{k=1}^{v_{1}} \beta_{k}^{-n_{k}}$ and

$$
d_{k}=\prod_{j=1}^{v_{1}}\left(\frac{\beta_{j}-\rho_{k}}{\beta_{j}}\right)^{n_{j}} \prod_{i=1, i \neq k}^{\mu_{1}} \frac{\rho_{i}}{\rho_{i}-\rho_{k}} \quad \text { for } 1 \leq k \leq \mu_{1} .
$$

In the following, we follow the convention that $\prod_{i=1, i \neq k}^{\mu_{1}}=1$ and $\prod_{m=1, m \neq j}^{\mu_{2}}=1$ in the $\mu_{1}=1$ and $\mu_{2}=1$ cases, respectively. We also need the following facts. 
Lemma 3.1. Suppose that $\mu_{1} \geq 1$ and $\mu_{2} \geq 1$. Then the following statements hold.

(a) For $i=1, \ldots, \mu_{1}$, we have

$$
-\lambda \sum_{k=1}^{v_{1}} \sum_{j=1}^{n_{k}} \frac{\left(\beta_{k}\right)^{j} c_{k j}}{\left(\beta_{k}-\rho_{i}\right)^{j}}-\mu \sum_{p=1}^{v_{2}} \sum_{m=1}^{\ell_{p}} \frac{\left(\alpha_{p}\right)^{m} \tilde{c}_{p m}}{\left(\alpha_{p}+\rho_{i}\right)^{m}}+(\mu+\lambda+r)-a \rho_{i}-\frac{b^{2} \rho_{i}^{2}}{2}=0,
$$

and, for $\xi=1, \ldots, \mu_{2}$, we have

$$
-\lambda \sum_{k=1}^{v_{1}} \sum_{j=1}^{n_{k}} \frac{\left(\beta_{k}\right)^{j} c_{k j}}{\left(\beta_{k}-\tilde{\rho_{\xi}}\right)^{j}}-\mu \sum_{p=1}^{v_{2}} \sum_{m=1}^{\ell_{p}} \frac{\left(\alpha_{p}\right)^{m} \tilde{c}_{p m}}{\left(\alpha_{p} \tilde{\rho}_{\xi}\right)^{m}}+(\mu+\lambda+r)-a \tilde{\rho_{\xi}}-\frac{b^{2} \tilde{\rho}_{\xi}^{2}}{2}=0 .
$$

(b) For $1 \leq k \leq v_{1}$ and $1 \leq \xi \leq n_{k}$, we obtain

$$
\sum_{j=1}^{\mu_{1}} \frac{d_{j} \rho_{j}}{\left(\beta_{k}-\rho_{j}\right)^{\xi}}= \begin{cases}d_{0} & \text { if } a<0, b=0, \text { and } \xi=1 \\ 0 & \text { otherwise. }\end{cases}
$$

(c) For $1 \leq p \leq v_{2}$ and $1 \leq m \leq \ell_{p}$, we obtain

$$
\sum_{j=1}^{\mu_{2}} \frac{\tilde{d}_{j} \tilde{\rho}_{j}}{\left(\alpha_{p}+\tilde{\rho}_{j}\right)^{m}}= \begin{cases}\tilde{d}_{0} & \text { if } a>0, b=0, \text { and } m=1, \\ 0 & \text { otherwise. }\end{cases}
$$

(d) For any complex numbers $A_{p, m}$ and $\omega$, we have

$$
\begin{gathered}
\sum_{\eta=1}^{\mu_{2}} \sum_{p=1}^{v_{2}} \sum_{m=1}^{\ell_{p}} \frac{A_{p, m} \tilde{d}_{\eta} \tilde{\rho}_{\eta}}{\omega-\tilde{\rho}_{\eta}}\left(\frac{1}{\left(\alpha_{p}+\tilde{\rho}_{\eta}\right)^{m}}-\frac{1}{\left(\alpha_{p}+\omega\right)^{m}}\right) \\
=\sum_{p=1}^{v_{2}} \sum_{m=1}^{\ell_{p}} \frac{A_{p, m}}{\left(\alpha_{p}+\omega\right)^{m}}\left(\tilde{d}_{0} \mathbf{1}_{\{a>0, b=0\}}\right) .
\end{gathered}
$$

(e) For any complex numbers $A_{s}$, we obtain

$$
\begin{aligned}
\sum_{s=1}^{\mu_{1}} \sum_{\eta=1}^{\mu_{2}} \frac{d_{s} \rho_{s} A_{s} \tilde{d}_{\eta} \tilde{\rho}_{\eta}}{\rho_{s}-\tilde{\rho}_{\eta}}\left[\sum_{k=1}^{v_{1}} \sum_{j=1}^{n_{k}} \frac{-\lambda\left(\beta_{k}\right)^{j} c_{k j}}{\left(\beta_{k}-\rho_{s}\right)^{j}}-a \rho_{s}-\frac{b^{2} \rho_{s}^{2}}{2}\right. \\
\left.-\left(\sum_{k=1}^{v_{1}} \sum_{j=1}^{n_{k}} \frac{-\lambda\left(\beta_{k}\right)^{j} c_{k j}}{\left(\beta_{k}-\tilde{\rho}_{\eta}\right)^{j}}-a \tilde{\rho}_{\eta}-\frac{b^{2} \tilde{\rho}_{\eta}^{2}}{2}\right)\right] \\
=\mathbf{1}_{\{a>0, b=0\}} \sum_{s=1}^{\mu_{1}} \sum_{p=1}^{v_{2}} \sum_{m=1}^{\ell_{p}} \frac{\left(-\tilde{d}_{0}\right) d_{s} \rho_{s} A_{s} \mu\left(\alpha_{p}\right)^{m} \tilde{c}_{p m}}{\left(\alpha_{p}+\rho_{s}\right)^{m}}
\end{aligned}
$$

(f) The following identities hold:

$$
\begin{gathered}
\frac{-a d_{0}}{r} \sum_{j=1}^{\mu_{2}} \tilde{d}_{j} \tilde{\rho}_{j}=1 \quad \text { if } a<0, b=0, \\
\frac{a \tilde{d}_{0}}{r} \sum_{j=1}^{\mu_{1}} d_{j} \rho_{j}=1 \quad \text { if } a>0, b=0,
\end{gathered}
$$


and

$$
\frac{1}{r} \sum_{s=1}^{\mu_{1}} \sum_{\eta=1}^{\mu_{2}} d_{s} \rho_{s} \tilde{d}_{\eta} \tilde{\rho}_{\eta}\left(\frac{b^{2}}{2}\right)=1 \quad \text { if } b \neq 0 .
$$

Proof. (a) The identities follow from the facts that $-\mathrm{i} \tilde{\rho}_{1}, \ldots,-\mathrm{i} \tilde{\rho}_{\mu_{2}}$ and $-\mathrm{i} \rho_{1}, \ldots,-\mathrm{i} \rho_{\mu_{1}}$ are solutions of $r-\psi(z)=0$ and (2.3).

(b) Since $m_{j}=1$ for $j=1, \ldots, \mu_{1}$, by Theorem 2.2, we obtain

$$
\psi_{r}^{+}(u)=\prod_{k=1}^{v_{1}}\left(\frac{u+\mathrm{i} \beta_{k}}{\mathrm{i} \beta_{k}}\right)^{n_{k}} \prod_{j=1}^{\mu_{1}} \frac{\mathrm{i} \rho_{j}}{u+\mathrm{i} \rho_{j}} .
$$

Note that if $a<0$ and $b=0$ then $\mu_{1}=\sum_{k=1}^{v_{1}} n_{k}$; otherwise, $\mu_{1}=\sum_{k=1}^{v_{1}} n_{k}+1$. Applying fractional decomposition to the right-hand side of (3.10) gives

$$
\prod_{k=1}^{v_{1}}\left(\frac{u+\mathrm{i} \beta_{k}}{\mathrm{i} \beta_{k}}\right)^{n_{k}} \prod_{j=1}^{\mu_{1}} \frac{\mathrm{i} \rho_{j}}{u+\mathrm{i} \rho_{j}}=\mathbf{1}_{\left\{\mu_{1} \geq 1\right\}} \sum_{j=1}^{\mu_{1}} \frac{d_{j} \rho_{j} \mathrm{i}}{u+\mathrm{i} \rho_{j}}+\mathbf{1}_{\{a<0, b=0\}} d_{0},
$$

where $\lim _{u \rightarrow-\mathrm{i} \rho_{j}}\left(u+\mathrm{i} \rho_{j}\right) \psi_{r}^{+}(u)=d_{j} \rho_{j} \mathrm{i}$ and the $d_{j}$ are given in (3.3). For $1 \leq k \leq v_{1}$ and $1 \leq \xi \leq n_{k}$, our results follow by differentiating both sides of (3.11) $\xi-1$ times at $u=-\mathrm{i} \beta_{k}$.

(c) Note that we have

$$
\psi_{r}^{-}(u)=\prod_{k=1}^{v_{2}}\left(\frac{u-\mathrm{i} \alpha_{k}}{-\mathrm{i} \alpha_{k}}\right)^{\ell_{k}} \prod_{j=1}^{\mu_{2}} \frac{\mathrm{i} \tilde{\rho}_{j}}{u+\mathrm{i} \tilde{\rho}_{j}} .
$$

Also, if $a>0$ and $b=0$ then $\mu_{2}=\sum_{k=1}^{v_{2}} \ell_{k}$; otherwise, $\mu_{2}=\sum_{k=1}^{v_{2}} \ell_{k}+1$. Applying fractional decomposition to the right-hand side of (3.12) gives

$$
\prod_{k=1}^{v_{2}}\left(\frac{u-\mathrm{i} \alpha_{k}}{-\mathrm{i} \alpha_{k}}\right)^{\ell_{k}} \prod_{j=1}^{\mu_{2}} \frac{\mathrm{i} \tilde{\rho}_{j}}{u+\mathrm{i} \tilde{\rho}_{j}}=\mathbf{1}_{\left\{\mu_{2} \geq 1\right\}} \sum_{j=1}^{\mu_{2}} \frac{\tilde{d}_{j} \tilde{\rho}_{j}(-\mathrm{i})}{u+\mathrm{i} \tilde{\rho}_{j}}+\mathbf{1}_{\{a>0, b=0\}} \tilde{d}_{0},
$$

where $\lim _{u \rightarrow-\mathrm{i} \tilde{\rho}_{j}}\left(u+\mathrm{i} \tilde{\rho}_{j}\right) \psi_{r}^{-}(u)=(-\mathrm{i}) \tilde{d}_{j} \tilde{\rho}_{j}$ and the $\tilde{d}_{j}$ are given in (3.1). For $1 \leq p \leq v_{2}$ and $1 \leq m \leq \ell_{p}$, our result follows by differentiating both sides of (3.13) $m-1$ times at $u=\mathrm{i} \alpha_{k}$.

(d) Observe that

$$
\sum_{q=1}^{m} \frac{1}{\left(\alpha_{p}+\tilde{\rho}_{\eta}\right)^{q}\left(\alpha_{p}+\omega\right)^{m-q+1}}=\frac{1}{\omega-\tilde{\rho}_{\eta}}\left(\frac{1}{\left(\alpha_{p}+\tilde{\rho}_{\eta}\right)^{m}}-\frac{1}{\left(\alpha_{p}+\omega\right)^{m}}\right) .
$$

Given any complex numbers $A_{p, m}$ and $w$, we have

$$
\begin{gathered}
\sum_{\eta=1}^{\mu_{2}} \sum_{p=1}^{v_{2}} \sum_{m=1}^{\ell_{p}} \frac{A_{p, m} \tilde{d}_{\eta} \tilde{\rho}_{\eta}}{\omega-\tilde{\rho}_{\eta}}\left(\frac{1}{\left(\alpha_{p}+\tilde{\rho}_{\eta}\right)^{m}}-\frac{1}{\left(\alpha_{p}+\omega\right)^{m}}\right) \\
=\sum_{\eta=1}^{\mu_{2}} \sum_{p=1}^{v_{2}} \sum_{m=1}^{\ell_{p}} \sum_{q=1}^{m} \frac{A_{p, m} \tilde{d}_{\eta} \tilde{\rho}_{\eta}}{\left(\alpha_{p}+\tilde{\rho}_{\eta}\right)^{q}(\alpha+\omega)^{m-q+1}}
\end{gathered}
$$




$$
\begin{aligned}
& =\sum_{p=1}^{v_{2}} \sum_{m=1}^{\ell_{p}} \sum_{q=1}^{m} \frac{A_{p, m}}{\left(\alpha_{p}+\omega\right)^{m-q+1}}\left(\sum_{\eta=1}^{\mu_{2}} \frac{\tilde{d}_{\eta} \tilde{\rho}_{\eta}}{\left(\alpha_{p}+\tilde{\rho}_{\eta}\right)^{q}}\right) \\
& =\sum_{p=1}^{v_{2}} \sum_{m=1}^{\ell_{p}} \frac{A_{p, m}}{\left(\alpha_{p}+\omega\right)^{m}}\left(\tilde{d}_{0} \mathbf{1}_{\{a>0, b=0\}}\right),
\end{aligned}
$$

where the last equality follows from (3.6).

(e) It is clear from (3.4) and (3.5) that

$$
\begin{aligned}
\sum_{s=1}^{\mu_{1}} \sum_{\eta=1}^{\mu_{2}} \frac{d_{s} \rho_{s} A_{s} \tilde{d}_{\eta} \tilde{\rho}_{\eta}}{\left(\rho_{s}-\tilde{\rho}_{\eta}\right)} & \\
\times & {\left[-\lambda \sum_{k=1}^{v_{1}} \sum_{j=1}^{n_{k}} \frac{\left(\beta_{k}\right)^{j} c_{k j}}{\left(\beta_{k}-\rho_{s}\right)^{j}}-\mu \sum_{p=1}^{v_{2}} \sum_{m=1}^{\ell_{p}} \frac{\left(\alpha_{p}\right)^{m} \tilde{c}_{p m}}{\left(\alpha_{p}+\rho_{s}\right)^{m}}-a \rho_{s}-\frac{b^{2} \rho_{s}^{2}}{2}\right.} \\
& \left.\quad-\left(-\lambda \sum_{k=1}^{v_{1}} \sum_{j=1}^{n_{k}} \frac{\left(\beta_{k}\right)^{j} c_{k j}}{\left(\beta_{k}-\tilde{\rho}_{\eta}\right)^{j}}-\mu \sum_{p=1}^{v_{2}} \sum_{m=1}^{\ell_{p}} \frac{\left(\alpha_{p}\right)^{m} \tilde{c}_{p m}}{\left(\alpha_{p}+\tilde{\rho}_{\eta}\right)^{m}}-a \tilde{\rho}_{\eta}-\frac{b^{2} \tilde{\rho}_{\eta}^{2}}{2}\right)\right] \\
= & 0 .
\end{aligned}
$$

This together with (3.7) yields (3.8).

(f) From Theorem 2.2, we have the following results.

$$
\begin{gathered}
\mu_{1}=\sum_{p=1}^{v_{1}} n_{p} \quad \text { and } \quad \mu_{2}=\sum_{m=1}^{v_{2}} \ell_{m}+1 \quad \text { if } a<0, b=0, \\
\mu_{1}=\sum_{p=1}^{v_{1}} n_{p}+1 \quad \text { and } \quad \mu_{2}=\sum_{m=1}^{v_{2}} \ell_{m} \quad \text { if } a>0, b=0, \\
\mu_{1}=\sum_{p=1}^{v_{1}} n_{p}+1 \quad \text { and } \quad \mu_{2}=\sum_{m=1}^{v_{2}} \ell_{m}+1 \quad \text { if } b \neq 0 .
\end{gathered}
$$

By applying the Wiener-Hopf factorization formula and combining (3.11) and (3.13), we see that, for $b=0$,

$$
\begin{aligned}
\frac{r}{r-\psi(u)}= & \frac{r \prod_{p=1}^{v_{1}}\left(u+\mathrm{i} \beta_{p}\right)^{n_{p}} \prod_{m=1}^{v_{2}}\left(u-\mathrm{i} \alpha_{m}\right)^{\ell_{m}}}{(-\mathrm{i} a) \prod_{j=1}^{\mu_{1}}\left(u+\mathrm{i} \rho_{j}\right) \prod_{k=1}^{\mu_{2}}\left(u+\mathrm{i} \tilde{\rho}_{k}\right)} \\
= & \left(\mathbf{1}_{\left\{\mu_{1} \geq 1\right\}} \sum_{j=1}^{\mu_{1}} \frac{d_{j} \rho_{j} \mathrm{i}}{u+\mathrm{i} \rho_{j}}+\mathbf{1}_{\{a<0, b=0\}} d_{0}\right) \\
& \times\left(\mathbf{1}_{\left\{\mu_{2} \geq 1\right\}} \sum_{j=1}^{\mu_{2}} \frac{\tilde{d}_{j} \tilde{\rho}_{j}(-\mathrm{i})}{u+\mathrm{i} \tilde{\rho}_{j}}+\mathbf{1}_{\{a>0, b=0\}} \tilde{d}_{0}\right),
\end{aligned}
$$


and, for $b \neq 0$,

$$
\begin{aligned}
\frac{r}{r-\psi(u)} & =\frac{r \prod_{p=1}^{v_{1}}\left(u+\mathrm{i} \beta_{p}\right)^{n_{p}} \prod_{m=1}^{v_{2}}\left(u-\mathrm{i} \alpha_{m}\right)^{\ell_{m}}}{\left(b^{2} / 2\right) \prod_{j=1}^{\mu_{1}}\left(u+\mathrm{i} \rho_{j}\right) \prod_{k=1}^{\mu_{2}}\left(u+\mathrm{i} \tilde{\rho}_{k}\right)} \\
& =\left(\sum_{j=1}^{\mu_{1}} \frac{d_{j} \rho_{j} \mathrm{i}}{u+\mathrm{i} \rho_{j}}\right)\left(\sum_{j=1}^{\mu_{2}} \frac{\tilde{d}_{j} \tilde{\rho}_{j}(-\mathrm{i})}{u+\mathrm{i} \tilde{\rho}_{j}}\right) .
\end{aligned}
$$

For the $b=0$ case, our results follow by multiplying both sides of (3.15) by $u$, letting $u \rightarrow \infty$, and using (3.14). For the $b \neq 0$ case, we obtain our result by multiplying both sides of (3.16) by $u^{2}$, letting $u \rightarrow \infty$, and using (3.14).

Observe that if $v_{1} \geq 1$ (i.e. there are upside jumps for the process $X$ ) then the function $\psi(\mathrm{i} z$ ) is a real analytic function in $\left(-\beta_{1}, 0\right)$ with $\psi(0)=0$ and $\lim _{z \downarrow-\beta_{1}} \psi(\mathrm{i} z)=\infty$. Hence, we have $0<\rho_{1}<\beta_{1}$.

Definition 3.1. We write $g \in \pi_{0}$ if the function $g: \mathbb{R} \rightarrow \mathbb{R}$ is absolutely continuous on every compact interval and there exist $A_{1}>0, A_{2}>0$, and $\theta \in\left(0, \rho_{1}\right)$ such that $|g(x)| \leq A_{1}+A_{2} \mathrm{e}^{\theta x}$ for all $x \in \mathbb{R}$.

For any $g \in \pi_{0}$, we define $Q_{g}(x)$ by

$$
\begin{gathered}
Q_{g}(x)=\mathbf{1}_{\left\{\mu_{2} \geq 1\right\}} \sum_{\eta=1}^{\mu_{2}} \frac{\tilde{d}_{\eta} \tilde{\rho}_{\eta}}{r}\left\{\sum_{k=1}^{v_{1}} \sum_{j=1}^{n_{k}} \sum_{\ell=1}^{j} \frac{-\lambda\left(\beta_{k}\right)^{j} c_{k j}}{\left(\beta_{k}-\tilde{\rho}_{\eta}\right)^{\ell}(j-\ell) !} \mathrm{e}^{\beta_{k} x}\right. \\
\quad \times \int_{x}^{\infty}(y-x)^{j-\ell} g(y) \mathrm{e}^{-\beta_{k} y} \mathrm{~d} y \\
\left.\quad-\left(\left(a+\frac{b^{2} \tilde{\rho}_{\eta}}{2}\right) g(x)+\frac{b^{2}}{2} g^{\prime}(x)\right)\right\} \\
+\frac{\tilde{d}_{0}}{r}\left\{\sum_{k=1}^{v_{1}} \sum_{j=1}^{n_{k}} \frac{-\lambda\left(\beta_{k}\right)^{j} c_{k j}}{(j-1) !} \mathrm{e}^{\beta_{k} x} \int_{x}^{\infty}(u-x)^{j-1} g(u) \mathrm{e}^{-\beta_{k} u} \mathrm{~d} u\right. \\
\left.+(\lambda+\mu+r) g(x)-a g^{\prime}(x)\right\} \mathbf{1}_{\{a>0, b=0\}} .
\end{gathered}
$$

We show below that $Q_{g}$ is a solution of the averaging problem (2.1).

Theorem 3.1. For any $g \in \pi_{0}$ and $r>0$, we have $\mathrm{E}\left[Q_{g}\left(M_{r}+x\right)\right]=g(x)$ for all $x \in \mathbb{R}$.

Proof. Observe that

$$
\mathrm{E} Q_{g}\left(M_{r}+x\right)=\int_{0}^{\infty} Q_{g}(y+x) f_{M}(y) \mathrm{d} y=\int_{x}^{\infty} Q_{g}(u) f_{M}(u-x) \mathrm{d} u
$$

where

$$
f_{M_{r}}(z)=\mathbf{1}_{\{a<0, b=0\}} d_{0} \delta_{0}(\mathrm{~d} z)+\mathbf{1}_{\left\{\mu_{1} \geq 1\right\}} \sum_{j=1}^{\mu_{1}} d_{j} \rho_{j} \mathrm{e}^{-\rho_{j} z} \mathbf{1}_{\{z>0\}} .
$$

We write

$$
\begin{aligned}
Q_{g}(x)= & \mathbf{1}_{\left\{\mu_{2} \geq 1\right\}}\left(Q_{g}^{(1)}(x)+Q_{g}^{(2)}(x)+Q_{g}^{(3)}(x)\right) \\
& +\mathbf{1}_{\{a>0, b=0\}}\left(Q_{g}^{(4)}(x)+Q_{g}^{(5)}(x)+Q_{g}^{(6)}(x)\right),
\end{aligned}
$$


where

$$
\begin{aligned}
Q_{g}^{(1)}(x) & =\sum_{\eta=1}^{\mu_{2}} \frac{\tilde{d}_{\eta} \tilde{\rho}_{\eta}}{r}\left\{\sum_{k=1}^{v_{1}} \sum_{j=1}^{n_{k}} \sum_{\ell=1}^{j} \frac{-\lambda\left(\beta_{k}\right)^{j} c_{k j}}{\left(\beta_{k}-\tilde{\rho}_{\eta}\right)^{\ell}(j-\ell) !} \mathrm{e}^{\beta_{k} x} \int_{x}^{\infty}(y-x)^{j-\ell} g(y) \mathrm{e}^{-\beta_{k} y} \mathrm{~d} y\right\} \\
Q_{g}^{(2)}(x) & =\left(-\sum_{\eta=1}^{\mu_{2}} \frac{\tilde{d}_{\eta} \tilde{\rho}_{\eta}}{r}\right) \frac{b^{2}}{2} g^{\prime}(x) \\
Q_{g}^{(3)}(x) & =-\sum_{\eta=1}^{\mu_{2}} \frac{\tilde{d}_{\eta} \tilde{\rho}_{\eta}}{r}\left(a+\frac{b^{2} \tilde{\rho}_{\eta}}{2}\right) g(x) \\
Q_{g}^{(4)}(x) & =\frac{\tilde{d}_{0}}{r}\left\{\sum_{k=1}^{v_{1}} \sum_{j=1}^{n_{k}} \frac{-\lambda\left(\beta_{k}\right)^{j} c_{k j}}{(j-1) !} \mathrm{e}^{\beta_{k} x} \int_{x}^{\infty}(u-x)^{j-1} g(u) \mathrm{e}^{-\beta_{k} u} \mathrm{~d} u\right\} \\
Q_{g}^{(5)}(x) & =\frac{\tilde{d}_{0}(-a)}{r} g^{\prime}(x) \\
\text { and } & \\
Q_{g}^{(6)}(x) & =\frac{\tilde{d}_{0}(\lambda+\mu+r)}{r} g(x)
\end{aligned}
$$

Taking account of $g \in \pi_{0}$ and using integration by parts, we obtain

$$
\begin{aligned}
\mathrm{e}^{\rho_{s} x} \int_{x}^{\infty} & \mathrm{e}^{\left(\beta_{k}-\rho_{s}\right) u} \int_{u}^{\infty}(y-u)^{j-\ell} g(y) \mathrm{e}^{-\beta_{k} y} \mathrm{~d} y \mathrm{~d} u \\
= & \frac{(j-\ell) ! \mathrm{e}^{\rho_{s} x}}{\left(\beta_{k}-\rho_{s}\right)^{j-\ell+1}} \int_{x}^{\infty} g(y) \mathrm{e}^{-\rho_{s} y} \mathrm{~d} y \\
& \quad+\sum_{\xi=1}^{j-\ell+1} \frac{-(j-\ell) ! \mathrm{e}^{\beta_{k} x}}{(j-\ell+1-\xi) !\left(\beta_{k}-\rho_{s}\right)^{\xi}} \int_{x}^{\infty}(y-x)^{j-\ell+1-\xi} g(y) \mathrm{e}^{-\beta_{k} y} \mathrm{~d} y .
\end{aligned}
$$

For simplicity, we also write

$$
I_{s, k, j-\ell}^{(1)}=\mathrm{e}^{\rho_{s} x} \int_{x}^{\infty} \mathrm{e}^{\left(\beta_{k}-\rho_{s}\right) u} \int_{u}^{\infty}(y-u)^{j-\ell} g(y) \mathrm{e}^{-\beta_{k} y} \mathrm{~d} y \mathrm{~d} u
$$

and

$$
I_{k, j-\ell+1-\xi}^{(2)}=\mathrm{e}^{\beta_{k} x} \int_{x}^{\infty}(y-x)^{j-\ell+1-\xi} g(y) \mathrm{e}^{-\beta_{k} y} \mathrm{~d} y .
$$

Using (3.18), (3.20), and (3.26), we obtain, for $\mu_{2} \geq 1$,

$$
\begin{aligned}
\int_{x}^{\infty} & Q_{g}^{(1)}(u) f_{M}(u-x) \mathrm{d} u \\
= & \mathbf{1}_{\left\{\mu_{1} \geq 1\right\}} \frac{1}{r}\left\{\sum_{s=1}^{\mu_{1}} \sum_{\eta=1}^{\mu_{2}} \sum_{k=1}^{v_{1}} \sum_{j=1}^{n_{k}} \sum_{\ell=1}^{j} \frac{d_{s} \rho_{s} \tilde{d}_{\eta} \tilde{\rho}_{\eta}(-\lambda)\left(\beta_{k}\right)^{j} c_{k j}}{\left(\beta_{k}-\tilde{\rho}_{\eta}\right)^{\ell}(j-\ell) !}\left(I_{s, k, j-\ell}^{(1)}\right)\right\} \\
& \quad+\mathbf{1}_{\{a<0, b=0\}} d_{0} Q_{g}^{(1)}(x)
\end{aligned}
$$




$$
\begin{aligned}
=\mathbf{1}_{\left\{\mu_{1} \geq 1\right\}} \frac{1}{r}\left\{\sum_{\eta=1}^{\mu_{2}} \sum_{k=1}^{v_{1}} \sum_{j=1}^{n_{k}} \sum_{\ell=1}^{j} \sum_{\xi=1}^{j-\ell+1}\right. & \frac{\tilde{d}_{\eta} \tilde{\rho}_{\eta} \lambda\left(\beta_{k}\right)^{j} c_{k j}}{\left(\beta_{k}-\tilde{\rho}_{\eta}\right)^{\ell}(j-\ell+1-\xi) !} \\
& \times\left(\sum_{s=1}^{\mu_{1}} \frac{d_{s} \rho_{s}}{\left(\beta_{k}-\rho_{s}\right)^{\xi}}\right) I_{k, j-\ell+1-\xi}^{(2)} \\
& +\mathbf{1}_{\left\{\mu_{1} \geq 1\right\}} \sum_{s=1}^{\mu_{1}} \sum_{\eta=1}^{\mu_{2}} \mathrm{~d}_{s} \rho_{s} \tilde{d}_{\eta} \tilde{\rho}_{\eta} \sum_{k=1}^{v_{1}} \sum_{j=1}^{n_{k}}\left(\sum_{\ell=1}^{j} \frac{-\lambda\left(\beta_{k}\right)^{j} c_{k j}}{\left(\beta_{k}-\tilde{\rho}_{\eta}\right)^{\ell}\left(\beta_{k}-\rho_{s}\right)^{j-\ell+1}}\right) \\
& \left.\quad \times \mathrm{e}^{\rho_{s} x} \int_{x}^{\infty} g(y) \mathrm{e}^{-\rho_{s} y} \mathrm{~d} y\right\}
\end{aligned}
$$

For $\mu_{1} \geq 1$, it follows from of Lemma 3.1(b) that, for $k=1, \ldots, v_{1}$ and $\xi=1, \ldots, n_{k}$,

$$
\sum_{j=1}^{\mu_{1}} \frac{d_{j} \rho_{j}}{\left(\beta_{k}-\rho_{j}\right)^{\xi}}= \begin{cases}\mathbf{1}_{\{\xi=1\}} d_{0} & \text { if } a<0 \text { and } b=0 \\ 0 & \text { otherwise. }\end{cases}
$$

This implies that, for $\mu_{2} \geq 1$,

$$
\begin{aligned}
\mathbf{1}_{\left\{\mu_{1} \geq 1\right\}} & \frac{1}{r} \sum_{\eta=1}^{\mu_{2}} \sum_{k=1}^{v_{1}} \sum_{j=1}^{n_{k}} \sum_{\ell=1}^{j} \sum_{\xi=1}^{j-\ell+1} \frac{\tilde{d}_{\eta} \tilde{\rho}_{\eta} \lambda\left(\beta_{k}\right)^{j} c_{k j}}{\left(\beta_{k}-\tilde{\rho}_{\eta}\right)^{\ell}(j-\ell+1-\xi) !}\left(\sum_{s=1}^{\mu_{1}} \frac{d_{s} \rho_{s}}{\left(\beta_{k}-\rho_{s}\right)^{\xi}}\right) I_{k, j-\ell+1-\xi}^{(2)} \\
& =-\mathbf{1}_{\{a<0, b=0\}} d_{0} Q_{g}^{(1)}(x) .
\end{aligned}
$$

By this and the identity

$$
\sum_{\ell=1}^{j} \frac{1}{\left(\beta_{k}-\tilde{\rho}_{\eta}\right)^{\ell}\left(\beta_{k}-\rho_{s}\right)^{j-\ell+1}}=\frac{1}{\rho_{s}-\tilde{\rho}_{\eta}}\left(\frac{1}{\left(\beta_{k}-\rho_{s}\right)^{j}}-\frac{1}{\left(\beta_{k}-\tilde{\rho}_{\eta}\right)^{j}}\right)
$$

(3.27) becomes

$$
\begin{aligned}
& \int_{x}^{\infty} Q_{g}^{(1)}(u) f_{M}(u-x) \mathrm{d} u \\
&=\mathbf{1}_{\left\{\mu_{1} \geq 1\right\}} \frac{1}{r}\left\{\sum_{s=1}^{\mu_{1}} \sum_{\eta=1}^{\mu_{2}} \frac{d_{s} \rho_{s} \tilde{d}_{\eta} \tilde{\rho}_{\eta}}{\rho_{s}-\tilde{\rho}_{\eta}}\left(\sum_{k=1}^{v_{1}} \sum_{j=1}^{n_{k}} \frac{-\lambda\left(\beta_{k}\right)^{j} c_{k j}}{\left(\beta_{k}-\rho_{s}\right)^{j}}+\frac{\lambda\left(\beta_{k}\right)^{j} c_{k j}}{\left(\beta_{k}-\tilde{\rho}_{\eta}\right)^{j}}\right) \mathrm{e}^{\rho_{s} x}\right. \\
&\left.\quad \times \int_{x}^{\infty} g(y) \mathrm{e}^{-\rho_{s} y} \mathrm{~d} y\right\}
\end{aligned}
$$

Again, by using integration by parts together with $g \in \pi_{0}$, we obtain

$$
\mathrm{e}^{\rho_{s} x} \int_{x}^{\infty} g^{\prime}(y) \mathrm{e}^{-\rho_{s} y} \mathrm{~d} y=-g(x)+\rho_{s} \mathrm{e}^{\rho_{s} x} \int_{x}^{\infty} g(y) \mathrm{e}^{-\rho_{s} y} \mathrm{~d} y .
$$


Combining (3.29) with (3.18) and (3.21) gives, for $\mu_{2} \geq 1$,

$$
\begin{aligned}
\int_{x}^{\infty} & Q_{g}^{(2)}(u) f_{M}(u-x) \mathrm{d} u \\
= & \mathbf{1}_{\left\{\mu_{1} \geq 1\right\}}\left(-\frac{b^{2}}{2 r}\right) \sum_{s=1}^{\mu_{1}} \sum_{\eta=1}^{\mu_{2}} d_{s} \rho_{s} \tilde{d}_{\eta} \tilde{\rho}_{\eta}\left(\mathrm{e}^{\rho_{s} x} \int_{x}^{\infty} g^{\prime}(y) \mathrm{e}^{-\rho_{s} y} \mathrm{~d} y\right) \\
= & \mathbf{1}_{\left\{\mu_{1} \geq 1\right\}}\left(-\frac{b^{2}}{2 r}\right) \sum_{s=1}^{\mu_{1}} \sum_{\eta=1}^{\mu_{2}} d_{s} \rho_{s} \tilde{d}_{\eta} \tilde{\rho}_{\eta}\left(-g(x)+\rho_{s} \mathrm{e}^{\rho_{s} x} \int_{x}^{\infty} g(y) \mathrm{e}^{-\rho_{s} y} \mathrm{~d} y\right) \\
= & \mathbf{1}_{\left\{\mu_{1} \geq 1\right\}} \frac{b^{2}}{2 r} g(x) \sum_{s=1}^{\mu_{1}} \sum_{\eta=1}^{\mu_{2}} d_{s} \rho_{s} \tilde{d}_{\eta} \tilde{\rho}_{\eta} \\
& -\mathbf{1}_{\left\{\mu_{1} \geq 1\right\}} \sum_{s=1}^{\mu_{1}} \sum_{\eta=1}^{\mu_{2}} \frac{d_{s} \rho_{s} \tilde{d}_{\eta} \tilde{\rho}_{\eta}}{\rho_{s}-\tilde{\rho}_{\eta}}\left(\frac{\rho_{s} b^{2}}{2 r}\left(\rho_{s}-\tilde{\rho}_{\eta}\right)\right) \mathrm{e}^{\rho_{s} x} \int_{x}^{\infty} g(y) \mathrm{e}^{-\rho_{s} y} \mathrm{~d} y .
\end{aligned}
$$

Also, using (3.18), (3.21), and (3.22) we have, for $\mu_{2} \geq 1$,

$$
\begin{aligned}
\int_{x}^{\infty} Q_{g}^{(3)}(u) f_{M}(u-x) \mathrm{d} u \\
=\mathbf{1}_{\left\{\mu_{1} \geq 1\right\}} \frac{(-1)}{r} \sum_{s=1}^{\mu_{1}} \sum_{\eta=1}^{\mu_{2}} d_{s} \rho_{s} \tilde{d}_{\eta} \tilde{\rho}_{\eta}\left(a+\frac{b^{2} \tilde{\rho}_{\eta}}{2}\right)\left(\mathrm{e}^{\rho_{s} x} \int_{x}^{\infty} g(y) \mathrm{e}^{-\rho_{s} y} \mathrm{~d} y\right) \\
\quad+\mathbf{1}_{\{a<0, b=0\}} d_{0} Q_{g}^{(3)}(x) \\
=\mathbf{1}_{\left\{\mu_{1} \geq 1\right\}} \frac{(-1)}{r} \sum_{s=1}^{\mu_{1}} \sum_{\eta=1}^{\mu_{2}} \frac{d_{s} \rho_{s} \tilde{d}_{\eta} \tilde{\rho}_{\eta}}{\rho_{s}-\tilde{\rho}_{\eta}}\left(a \rho_{s}+\frac{b^{2} \tilde{\rho}_{\eta} \rho_{s}}{2}-\left(a \tilde{\rho}_{\eta}+\frac{b^{2} \tilde{\rho}_{\eta}^{2}}{2}\right)\right) \mathrm{e}^{\rho_{s} x} \\
\quad \times \int_{x}^{\infty} g(y) \mathrm{e}^{-\rho_{s} y} \mathrm{~d} y \\
\quad+\mathbf{1}_{\{a<0, b=0\}} d_{0} Q_{g}^{(3)}(x) .
\end{aligned}
$$

Combining (3.28), (3.30), and (3.31) gives, for $\mu_{2} \geq 1$,

$$
\begin{aligned}
\mathrm{E}\left[\left(Q_{g}^{(1)}+\right.\right. & \left.\left.Q_{g}^{(2)}+Q_{g}^{(3)}\right)\left(M_{r}+x\right)\right] \\
= & \mathbf{1}_{\left\{\mu_{1} \geq 1\right\}} \frac{1}{r} \sum_{s=1}^{\mu_{1}} \sum_{\eta=1}^{\mu_{2}} \frac{d_{s} \rho_{s} \tilde{d}_{\eta} \tilde{\rho}_{\eta}}{\left(\rho_{s}-\tilde{\rho}_{\eta}\right)} \mathrm{e}^{\rho_{s} x} \int_{x}^{\infty} g(y) \mathrm{e}^{-\rho_{s} y} \mathrm{~d} y \\
\times & {\left[-\lambda \sum_{k=1}^{v_{1}} \sum_{j=1}^{n_{k}} \frac{\left(\beta_{k}\right)^{j} c_{k j}}{\left(\beta_{k}-\rho_{s}\right)^{j}}-a \rho_{s}-\frac{b^{2} \rho_{s}^{2}}{2}\right.} \\
& \left.-\left(-\lambda \sum_{k=1}^{v_{1}} \sum_{j=1}^{n_{k}} \frac{\left(\beta_{k}\right)^{j} c_{k j}}{\left(\beta_{k}-\tilde{\rho}_{\eta}\right)^{j}}-a \tilde{\rho}_{\eta}-\frac{b^{2} \tilde{\rho}_{\eta}^{2}}{2}\right)\right] \\
+ & \mathbf{1}_{\{a<0, b=0\}} d_{0} Q_{g}^{(3)}(x)+\mathbf{1}_{\left\{\mu_{1} \geq 1\right\}} \frac{b^{2}}{2 r} g(x) \sum_{s=1}^{\mu_{1}} \sum_{\eta=1}^{\mu_{2}} d_{s} \rho_{s} \tilde{d}_{\eta} \tilde{\rho}_{\eta} .
\end{aligned}
$$


Using Lemma 3.1(e), we obtain, for $\mu_{2} \geq 1$,

$$
\begin{aligned}
& \mathrm{E}\left[\left(Q_{g}^{(1)}+Q_{g}^{(2)}+Q_{g}^{(3)}\right)\left(M_{r}+x\right)\right] \\
& =\mathbf{1}_{\{a<0, b=0\}} d_{0} Q_{g}^{(3)}(x)+\mathbf{1}_{\left\{\mu_{1} \geq 1\right\}} \frac{b^{2}}{2 r} g(x) \sum_{s=1}^{\mu_{1}} \sum_{\eta=1}^{\mu_{2}} d_{s} \rho_{s} \tilde{d}_{\eta} \tilde{\rho}_{\eta} \\
& \quad+\mathbf{1}_{\left\{\mu_{1} \geq 1, a>0, b=0\right\}} \sum_{s=1}^{\mu_{1}} \sum_{p=1}^{v_{2}} \sum_{m=1}^{\ell_{p}} \frac{\left(-\tilde{d}_{0}\right) d_{s} \rho_{s} \mu\left(\alpha_{p}\right)^{m} \tilde{c}_{p m}}{r\left(\alpha_{p}+\rho_{s}\right)^{m}} \mathrm{e}^{\rho_{s} x} \int_{x}^{\infty} g(y) \mathrm{e}^{-\rho_{s} y} \mathrm{~d} y .
\end{aligned}
$$

Consequently, using (3.14), (3.9), and (3.19), we see that, for the $b \neq 0$ case,

$\mathrm{E}\left[Q_{g}\left(M_{r}+x\right)\right]=\mathrm{E}\left[\left(Q_{g}^{(1)}+Q_{g}^{(2)}+Q_{g}^{(3)}\right)\left(M_{r}+x\right)\right]=g(x)\left(\frac{b^{2}}{2 r} \sum_{s=1}^{\mu_{1}} \sum_{\eta=1}^{\mu_{2}} d_{s} \rho_{s} \tilde{d}_{\eta} \tilde{\rho}_{\eta}\right)=g(x)$

and, for the case in which $a<0$ and $b=0$,

$$
\mathrm{E}\left[Q_{g}\left(M_{r}+x\right)\right]=\mathrm{E}\left[\left(Q_{g}^{(1)}+Q_{g}^{(2)}+Q_{g}^{(3)}\right)\left(M_{r}+x\right)\right]=g(x)\left(\frac{(-a) d_{0}}{r} \sum_{\eta=1}^{\mu_{2}} \tilde{d}_{\eta} \tilde{\rho}_{\eta}\right)=g(x) .
$$

It remains to consider the case in which $a>0$ and $b=0$. By (3.14), we have $\mu_{1} \geq 1$. It follows from (3.18), (3.23), and (3.26) that

$$
\begin{aligned}
\int_{x}^{\infty} & Q_{g}^{(4)}(u) f_{M}(u-x) \mathrm{d} u \\
= & \sum_{k=1}^{v_{1}} \sum_{j=1}^{n_{k}} \sum_{\xi=1}^{j} \frac{\tilde{d}_{0} \lambda\left(\beta_{k}\right)^{j} c_{k j} \mathrm{e}^{\beta_{k} x}}{r(j-\xi) !}\left(\sum_{s=1}^{\mu_{1}} \frac{d_{s} \rho_{s}}{\left(\beta_{k}-\rho_{s}\right)^{\xi}}\right) \int_{x}^{\infty}(y-x)^{j-\xi} g(y) \mathrm{e}^{-\beta_{k} y} \mathrm{~d} y \\
& +\sum_{s=1}^{\mu_{1}} \sum_{k=1}^{v_{1}} \sum_{j=1}^{n_{k}} \frac{\tilde{d}_{0} d_{s} \rho_{s}(-\lambda)\left(\beta_{k}\right)^{j} c_{k j} \mathrm{e}^{\rho_{s} x}}{r\left(\beta_{k}-\rho_{s}\right)^{j}} \int_{x}^{\infty} g(y) \mathrm{e}^{-\rho_{s} y} \mathrm{~d} y \\
= & \sum_{s=1}^{\mu_{1}} \sum_{k=1}^{v_{1}} \sum_{j=1}^{n_{k}} \frac{\tilde{d}_{0} d_{s} \rho_{s}(-\lambda)\left(\beta_{k}\right)^{j} c_{k j} \mathrm{e}^{\rho_{s} x}}{r\left(\beta_{k}-\rho_{s}\right)^{j}} \int_{x}^{\infty} g(y) \mathrm{e}^{-\rho_{s} y} \mathrm{~d} y .
\end{aligned}
$$

The last equality follows from Lemma 3.1(b). Using (3.29), (3.18), and (3.24) we have

$$
\int_{x}^{\infty} Q_{g}^{(5)}(u) f_{M}(u-x) \mathrm{d} u=\left(\frac{-a \tilde{d}_{0}}{r}\right) \sum_{s=1}^{\mu_{1}} d_{s} \rho_{s}\left(-g(x)+\rho_{s} \mathrm{e}^{\rho_{s} x} \int_{x}^{\infty} g(y) \mathrm{e}^{-\rho_{s} y} \mathrm{~d} y\right) .
$$

By (3.18), (3.24), and (3.25), we have

$$
\int_{x}^{\infty} Q_{g}^{(6)}(u) f_{M}(u-x) \mathrm{d} u=\frac{\tilde{d}_{0}(\lambda+\mu+r)}{r} \sum_{s=1}^{\mu_{1}} d_{s} \rho_{s} \mathrm{e}^{\rho_{s} x} \int_{x}^{\infty} g(y) \mathrm{e}^{-\rho_{s} y} \mathrm{~d} y .
$$


It follows, from (3.19), (3.32), (3.33), (3.34), and (3.35), that

$$
\begin{aligned}
& \mathrm{E}\left[Q_{g}\left(M_{r}+x\right)\right] \\
& =\mathbf{1}_{\{a>0, b=0\}} \sum_{s=1}^{\mu_{1}} \frac{\tilde{d}_{0} d_{s} \rho_{s} \mathrm{e}^{\rho_{s} x}}{r} \int_{x}^{\infty} g(y) \mathrm{e}^{-\rho_{s} y} \mathrm{~d} y \\
& +\mathbf{1}_{\{a>0, b=0\}} \frac{\tilde{d}_{0} a}{r}\left(\sum_{j=1}^{\mu_{1}} d_{j} \rho_{j}\right) g(x) \\
& \times\left[-\lambda \sum_{k=1}^{v_{1}} \sum_{j=1}^{n_{k}} \frac{\left(\beta_{k}\right)^{j} c_{k j}}{\left(\beta_{k}-\rho_{s}\right)^{j}}-\mu \sum_{p=1}^{v_{2}} \sum_{m=1}^{\ell_{p}} \frac{\left(\alpha_{p}\right)^{m} \tilde{c}_{p m}}{\left(\alpha_{p}+\rho_{s}\right)^{m}}+(\mu+\lambda+r)-a \rho_{s}\right] .
\end{aligned}
$$

By Lemma 3.1(a) and (f), we obtain $\mathrm{E}\left[Q_{g}\left(M_{r}+x\right)\right]=g(x)$. The proof is complete.

Remark 3.1. (a) We write $g \in \mathcal{R}$ if $g: \mathbb{R} \rightarrow \mathbb{R}$ is an $L_{1}$-integrable function such that the Fourier transform $\hat{g}$, defined by $\hat{g}(\omega)=\int_{-\infty}^{\infty} \mathrm{e}^{-\mathrm{i} \omega x} g(x) \mathrm{d} x$, satisfies the integrability condition $\int_{-\infty}^{\infty}\left(1+|\omega|^{3}\right)|\hat{g}(\omega)| \mathrm{d} \omega<\infty$. As noted in [13], the set $\mathcal{R}$ belongs to the class of $\mathcal{C}_{b}^{3}$. This implies that every element in $\mathcal{R}$ is also in $\pi_{0}$. Surya [13] showed that, if $g \in \mathcal{R}$, the function $(1 / 2 \pi) \int_{-\infty}^{\infty}\left(\mathrm{e}^{\mathrm{i} \omega x} \hat{g}(\omega) / \psi_{r}^{-}(\omega)\right) \mathrm{d} \omega$ solves the American put-type averaging problem. By using the Fourier inversion formula, we can verify by direct calculation that, for $g \in \mathcal{R}$, the function $(1 / 2 \pi) \int_{-\infty}^{\infty}\left(\mathrm{e}^{\mathrm{i} \omega x} \hat{g}(\omega) / \psi_{r}^{+}(\omega)\right) \mathrm{d} \omega$ coincides with $Q_{g}(x)$. Moreover, we have the following identity

$$
\begin{aligned}
\left(\psi_{r}^{(+)}(\omega)\right)^{-1}= & \mathbf{1}_{\{a>0, b=0\}} \frac{\tilde{d}_{0}}{r}\left[\sum_{k=1}^{v_{1}} \sum_{j=1}^{n_{k}} \frac{-\lambda\left(\beta_{k}\right)^{j} c_{k j}}{\left(\beta_{k}-\mathrm{i} \omega\right)^{j}}-\mathrm{i} a \omega+(\lambda+\mu+r)\right] \\
+ & \mathbf{1}_{\left\{\mu_{2} \geq 1\right\}} \frac{1}{r} \sum_{\eta=1}^{\mu_{2}} \frac{\tilde{d}_{\eta} \tilde{\rho}_{\eta}}{\mathrm{i} \omega-\tilde{\rho}_{\eta}}\left[\left(\mathrm{i} \omega-\tilde{\rho}_{\eta}\right)\left(\frac{-\mathrm{i} \omega b^{2}}{2}-a-\frac{b^{2} \tilde{\rho}_{\eta}}{2}\right)\right. \\
& \left.+\left(\sum_{k=1}^{v_{1}} \sum_{j=1}^{n_{k}} \frac{-\lambda\left(\beta_{k}\right)^{j} c_{k j}}{\left(\beta_{k}-\mathrm{i} \omega\right)^{j}}+\frac{\lambda\left(\beta_{k}\right)^{j} c_{k j}}{\left(\beta_{k}-\tilde{\rho}_{\eta}\right)^{j}}\right)\right] .
\end{aligned}
$$

(b) It is interesting to note that if $g(x)=\sum_{m=1}^{M} h_{m} \mathrm{e}^{\theta_{m} x}$ with $0 \leq \max \left\{\theta_{m}: 1 \leq m \leq M\right\}<\rho_{1}$ then

$$
Q_{g}(x)=\sum_{m=1}^{M} h_{m} \mathrm{e}^{\theta_{m} x}\left(\psi_{r}^{+}\left(-\mathrm{i} \theta_{m}\right)\right)^{-1} .
$$

The result is consistent with those in [4], [10], and [13].

In the following we study some properties of $Q_{g}(x)$. We write $g \in \hat{\pi}_{0}$ if $g \in \pi_{0}$ is nondecreasing, $\{g>0\}=(\hat{a},+\infty)$ for some $\hat{a}<\infty$, and $g \in C^{1}(\hat{a},+\infty)$. In the remainder of the paper, we consider the model $X$ with $c_{k j}>0, \beta_{k}>0$, and $\alpha_{p}>0$ for $1 \leq k \leq v_{1}, 1 \leq$ $j \leq n_{k}$, and $1 \leq p \leq v_{2}$.

Proposition 3.1. Consider the reward function $g \in \hat{\pi}_{0}$ with $\{g>0\}=(\hat{a}, \infty)$ and $Q_{g}$ given by (3.17). Then the following statements hold.

(a) If there exists $\alpha>0$ such that $\lim _{x \rightarrow \infty} Q_{g}(x) \geq \alpha$ then there exists $x^{*}>\hat{a}$ such that $Q_{g}\left(x^{*}\right)=0$. 
(b) If $(g(u+x) / g(x))^{\prime}<0$ and $\left(g^{\prime}(x) / g(x)\right)^{\prime}<0$ for any $x>\hat{a}$ and $u>0$, then there exists at most one $x^{*} \in(\hat{a},+\infty)$ such that $Q_{g}\left(x^{*}\right)=0$.

(c) If both conditions in (a) and (b) hold, then there exists a unique $x^{*} \in(\hat{a},+\infty)$ such that $Q_{g}\left(x^{*}\right)=0$. Moreover, $Q_{g}(x)$ is increasing for $x>x^{*}$ and $Q_{g}(x)<0$ for $\hat{a}<x<x^{*}$.

Proof. Observe that

$$
\begin{aligned}
Q_{g}(x)= & \sum_{k=1}^{v_{1}} \sum_{j=1}^{n_{k}} \sum_{\ell=1}^{j} \frac{-\lambda\left(\beta_{k}\right)^{j} c_{k j}}{r(j-\ell) !}\left[\left(\mathbf{1}_{\left\{\mu_{2} \geq 1\right\}} \sum_{\eta=1}^{\mu_{2}} \frac{\tilde{d}_{\eta} \tilde{\rho}_{\eta}}{\left(\beta_{k}-\tilde{\rho}_{\eta}\right)^{\ell}}+\mathbf{1}_{\{a>0, b=0, \ell=1\}} \tilde{d}_{0}\right)\right. \\
& \left.\times \int_{0}^{\infty} u^{j-\ell} g(u+x) \mathrm{e}^{-\beta_{k} u} \mathrm{~d} u\right] \\
& -\left[\mathbf{1}_{\left\{\mu_{2} \geq 1\right\}} \frac{b^{2}}{2 r}\left(\sum_{\eta=1}^{\mu_{2}} \tilde{d}_{\eta} \tilde{\rho}_{\eta}\right)+\mathbf{1}_{\{a>0, b=0\}} \frac{a \tilde{d}_{0}}{r}\right] g^{\prime}(x) \\
& -\left[\mathbf{1}_{\left\{\mu_{2} \geq 1\right\}} \sum_{\eta=1}^{\mu_{2}} \frac{\tilde{d}_{\eta} \tilde{\rho}_{\eta}\left(a+b^{2} \tilde{\rho}_{\eta} / 2\right)}{r}-\mathbf{1}_{\{a>0, b=0\}} \frac{\tilde{d}_{0}}{r}(\lambda+\mu+r)\right] g(x) .
\end{aligned}
$$

We first show that $\lim _{t \rightarrow \hat{a}^{+}} Q_{g}(t)<0$. To do this, we first claim that, for $\mu_{2} \geq 1$ and $b \neq 0$,

$$
\sum_{\eta=1}^{\mu_{2}} \tilde{d}_{\eta} \tilde{\rho}_{\eta}=\frac{\prod_{j=1}^{\mu_{2}}-\tilde{\rho}_{j}}{\prod_{k=1}^{v_{2}}\left(\alpha_{k}\right)^{\ell_{k}}}>0
$$

and, for $a>0$ and $b=0$,

$$
\tilde{d}_{0}=\frac{\prod_{j=1}^{\mu_{2}}-\tilde{\rho}_{j}}{\prod_{k=1}^{v_{2}}\left(\alpha_{k}\right)^{\ell_{k}}}>0 .
$$

Also, we will show that, for $1 \leq k \leq v_{1}$ and $1 \leq \ell \leq n_{k}$,

$$
\mathbf{1}_{\left\{\mu_{2} \geq 1\right\}} \sum_{\eta=1}^{\mu_{2}} \frac{\tilde{d}_{\eta} \tilde{\rho}_{\eta}}{\left(\beta_{k}-\tilde{\rho}_{\eta}\right)^{\ell}}+\mathbf{1}_{\{a>0, b=0\}} \mathbf{1}_{\{\ell=1\}} \tilde{d}_{0}>0 .
$$

From identities (3.12) and (3.13), we obtain

$$
\mathrm{E}\left[\mathrm{e}^{u I_{r}}\right]=\prod_{k=1}^{v_{2}}\left(\frac{u+\alpha_{k}}{\alpha_{k}}\right)^{\ell_{k}} \prod_{j=1}^{\mu_{2}} \frac{-\tilde{\rho}_{j}}{u-\tilde{\rho}_{j}}=\mathbf{1}_{\left\{\mu_{2} \geq 1\right\}} \sum_{j=1}^{\mu_{2}} \frac{\tilde{d}_{j} \tilde{\rho}_{j}}{u-\tilde{\rho}_{j}}+\mathbf{1}_{\{a>0, b=0\}} \tilde{d}_{0} .
$$

We obtain (3.37) by multiplying both sides by $u$, letting $u \rightarrow \infty$ in (3.40), and using the fact that $\mu_{2}=\sum_{k=1}^{v_{2}} \ell_{k}+1$. Similarly, (3.38) follows by letting $u \rightarrow \infty$ in (3.40) and using the fact that $\mu_{2}=\sum_{k=1}^{v_{2}} \ell_{k}$. To verify (3.39), we note that differentiating both sides of (3.40) $\xi$ times at $u=\beta_{k}$ implies that

$$
\mathrm{E}\left[\left(I_{r}\right)^{\xi} \mathrm{e}^{\beta_{k} I_{r}}\right]=\mathbf{1}_{\left\{\mu_{2} \geq 1\right\}}(-1)^{\xi} \sum_{\eta=1}^{\mu_{2}} \frac{\tilde{d}_{\eta} \tilde{\rho}_{\eta}}{\left(\beta_{k}-\tilde{\rho}_{\eta}\right)^{\xi+1}}+\mathbf{1}_{\{a>0, b=0\}} \mathbf{1}_{\{\xi=0\}} \tilde{d}_{0} .
$$

This yields (3.39). Using (3.37)-(3.39) and (3.36), we obtain $\lim _{t \rightarrow \hat{a}^{+}} Q_{g}(t)<0$. 
To prove (a), note that, by the assumption in (a), we have $\lim _{x \rightarrow+\infty} Q_{g}(x)>0$ and $Q_{g}(x) \in$ $C(\hat{a},+\infty)$. These together with $\lim _{t \rightarrow \hat{a}^{+}} Q_{g}(t)<0$ and the intermediate value theorem, imply that there exists at least one $x^{*}$ in $(\hat{a}, \infty)$ such that $Q_{g}\left(x^{*}\right)=0$.

To prove (b), we write $Q_{g}(x)=g(x) h(x)$ for $x \in(\hat{a}, \infty)$, where

$$
\begin{aligned}
h(x)= & -\left[\mathbf{1}_{\left\{\mu_{2} \geq 1\right\}} \frac{b^{2}}{2 r}\left(\sum_{\eta=1}^{\mu_{2}} \tilde{d}_{\eta} \tilde{\rho}_{\eta}\right)+\mathbf{1}_{\{a>0, b=0\}} \frac{a \tilde{d}_{0}}{r}\right] \frac{g^{\prime}(x)}{g(x)} \\
& +\sum_{k=1}^{v_{1}} \sum_{j=1}^{n_{k}} \sum_{\ell=1}^{j} \frac{-\lambda\left(\beta_{k}\right)^{j} c_{k j}}{r(j-\ell) !}\left[\left(\mathbf{1}_{\left\{\mu_{2} \geq 1\right\}} \sum_{\eta=1}^{\mu_{2}} \frac{\tilde{d}_{\eta} \tilde{\rho}_{\eta}}{\left(\beta_{k}-\tilde{\rho}_{\eta}\right)^{\ell}}+\mathbf{1}_{\{a>0, b=0\}} \mathbf{1}_{\{\ell=1\}} \tilde{d}_{0}\right)\right. \\
& \left.\times \int_{0}^{\infty} u^{j-\ell} \frac{g(u+x)}{g(x)} \mathrm{e}^{-\beta_{k} u} \mathrm{~d} u\right] \\
& -\left[\mathbf{1}_{\left\{\mu_{2} \geq 1\right\}} \sum_{\eta=1}^{\mu_{2}} \frac{\tilde{d}_{\eta} \tilde{\rho}_{\eta}}{r}\left(a+\frac{b^{2} \tilde{\rho}_{\eta}}{2}\right)-\mathbf{1}_{\{a>0, b=0\}} \frac{\tilde{d}_{0}}{r}(\lambda+\mu+r)\right] .
\end{aligned}
$$

Taking account of (3.37)-(3.39), and the conditions $(g(u+x) / g(x))^{\prime}<0$ and $\left(g^{\prime}(x) / g(x)\right)^{\prime}<0$ for any $x>\hat{a}$ and $u>0$, we see that $h^{\prime}(x)>0$ for any $x>\hat{a}$. This implies that there exists at most one $x^{*} \in(\hat{a}, \infty)$ such that $h\left(x^{*}\right)=0$. Hence, $Q_{g}(x)=0$ has at most one solution in $(\hat{a},+\infty)$.

To prove (c), by (a) and (b) we see that there exists only one $x^{*} \in(\hat{a}, \infty)$ such that $Q_{g}\left(x^{*}\right)=$ 0 . Furthermore, since $\lim _{t \rightarrow \hat{a}^{+}} Q_{g}(t)<0, Q_{g}$ is continuous on $(\hat{a}, \infty)$ and $Q_{g}(x)=0$ has a unique solution on $(\hat{a}, \infty)$, we have $Q_{g}(x)<0$ for $x \in\left(\hat{a}, x^{*}\right)$. For $x>x^{*}$, we have $Q_{g}(x)=g(x) h(x)$ and, hence, $Q_{g}^{\prime}(x)=g^{\prime}(x) h(x)+g(x) h^{\prime}(x)$. Since each term on the righthand side is nonnegative, and $g(x)$ and $h^{\prime}(x)$ are positive, we obtain $Q_{g}^{\prime}(x)>0$ for $x>x^{*}$ and, hence, $Q_{g}(x)$ is increasing on $\left(x^{*}, \infty\right)$.

Combining Theorem 2.1, Theorem 3.1, and Proposition 3.1 gives the following main result.

Theorem 3.2. Consider a reward function $g(x) \in \hat{\pi}_{0}$ with $\{g>0\}=(\hat{a}, \infty)$ and $Q_{g}(x)$ given by (3.17). Assume that the following conditions hold.

(a) There exists $\alpha>0$ such that $\lim _{x \rightarrow \infty} Q_{g}(x) \geq \alpha$.

(b) $(g(u+x) / g(x))^{\prime}<0$ and $\left(g^{\prime}(x) / g(x)\right)^{\prime}<0$ for any $x>\hat{a}$ and $u>0$.

Then the value function for the optimal stopping problem (1.1) is given by

$$
V(x)=\mathrm{E}_{x}\left(\mathrm{e}^{-r \tau^{*}} g\left(X_{\tau^{*}}\right)\right)=\int_{x^{*}-x}^{\infty} Q_{g}(x+m) f_{M_{r}}(m) \mathrm{d} m .
$$

Here $x^{*}$ is the unique solution of the equation $Q_{g}(x)=0$ in $(\hat{a}, \infty), \tau^{*}=\inf \left\{t>0: X_{t}>x^{*}\right\}$, and $f_{M_{r}}$ is given by (3.2).

\section{Examples}

In the following, we first reproduce the results of Kyprianou and Surya [8], Novikov and Shiryaev [12], and Deligiannidis et al. [6]. 
Example 4.1. (Option with power function.) Consider the optimal stopping problem (1.1) with $g(x)=\left(x^{+}\right)^{\gamma}, \gamma>1$. According to (3.17), $Q_{g}(x)$ is given by

$$
\begin{aligned}
& Q_{g}(x)=\mathbf{1}_{\left\{\mu_{2} \geq 1\right\}} \sum_{\eta=1}^{\mu_{2}} \frac{\tilde{d}_{\eta} \tilde{\rho}_{\eta}}{r}\left[\sum_{k=1}^{v_{1}} \sum_{j=1}^{n_{k}} \sum_{\ell=1}^{j} \frac{-\lambda\left(\beta_{k}\right)^{j} c_{k j}}{\left(\beta_{k}-\tilde{\rho}_{\eta}\right)^{\ell}(j-\ell) !} \int_{0}^{\infty} u^{j-\ell} \mathrm{e}^{-\beta_{k} u}(u+x)^{\gamma} \mathrm{d} u\right. \\
&\left.\quad-\left(\left(a+\frac{b^{2} \tilde{\rho}_{\eta}}{2}\right) x^{\gamma}+\frac{b^{2}}{2} \gamma x^{\gamma-1}\right)\right] \\
&+ \mathbf{1}_{\{a>0, b=0\}} \frac{\tilde{d}_{0}}{r}\left\{\sum_{k=1}^{v_{1}} \sum_{j=1}^{n_{k}} \frac{-\lambda\left(\beta_{k}\right)^{j} c_{k j}}{(j-1) !} \int_{0}^{\infty} u^{j-1} \mathrm{e}^{-\beta_{k} u}(u+x)^{\gamma} \mathrm{d} u\right. \\
&\left.+(\lambda+\mu+r) x^{\gamma}-a \gamma x^{\gamma-1}\right\} .
\end{aligned}
$$

Moreover, we have

$$
\begin{aligned}
\lim _{x \rightarrow \infty} Q_{g}(x) x^{-\gamma}= & \mathbf{1}_{\left\{\mu_{2} \geq 1\right\}} \sum_{\eta=1}^{\mu_{2}} \frac{\tilde{d}_{\eta} \tilde{\rho}_{\eta}}{r}\left\{\sum_{k=1}^{v_{1}} \sum_{j=1}^{n_{k}} \sum_{\ell=1}^{j} \frac{-\lambda\left(\beta_{k}\right)^{j} c_{k j}}{\left(\beta_{k}-\tilde{\rho}_{\eta}\right)^{\ell} \beta_{k}^{j-\ell+1}}-\left(a+\frac{b^{2} \tilde{\rho}_{\eta}}{2}\right)\right\} \\
& +\mathbf{1}_{\{a>0, b=0\}} \frac{\tilde{d}_{0}}{r}(\mu+r) .
\end{aligned}
$$

By using the identity

$$
\sum_{k=1}^{v_{1}} \sum_{j=1}^{n_{k}}\left(\sum_{\ell=1}^{j} \frac{-\lambda\left(\beta_{k}\right)^{j} c_{k j}}{\left(\beta_{k}-\tilde{\rho}_{\eta}\right)^{\ell}\left(\beta_{k}-\theta\right)^{j-\ell+1}}\right)=\frac{1}{\theta-\tilde{\rho}_{\eta}}\left(\sum_{k=1}^{v_{1}} \sum_{j=1}^{n_{k}} \frac{-\lambda\left(\beta_{k}\right)^{j} c_{k j}}{\left(\beta_{k}-\theta\right)^{j}}+\frac{\lambda\left(\beta_{k}\right)^{j} c_{k j}}{\left(\beta_{k}-\tilde{\rho}_{\eta}\right)^{j}}\right)
$$

and Remark 3.1(a), we see that $\lim _{x \rightarrow \infty} Q_{g}(x) x^{-\gamma}=\left(\psi_{r}^{+}(0)\right)^{-1}=1$. Also, observe that, for $x>0$ and $u>0,(g(u+x) / g(x))^{\prime}=\left((1+u / x)^{\gamma}\right)^{\prime}=\gamma(1+u / x)^{\gamma-1}\left(-u / x^{2}\right)<0$ and $\left(g^{\prime}(x) / g(x)\right)^{\prime}=\left(\gamma x^{-1}\right)^{\prime}=-\gamma x^{-2}<0$. By Theorem 3.2, there exists a unique $x^{*}$ such that $Q_{g}\left(x^{*}\right)=0$ and $\tau^{*}=\inf \left\{t \geq 0: X_{t} \geq x^{*}\right\}$ is the optimal stopping time for the optimal stopping problem (1.1) with $g(x)=\left(x^{+}\right)^{\gamma}, \gamma>1$.

Remark 4.1. (a) Assume that $g(x)=\left(x^{+}\right)^{n}$, where $n \in \mathbb{N} \cup\{0\}$. Write $Q_{n}(x)=Q_{g}(x)$. Direct calculations show that $Q_{n}(x)$ satisfies $Q_{0}(x)=1, \mathrm{~d} Q_{n}(x) / \mathrm{d} x=n Q_{n-1}(x)$, and $\mathrm{E}\left[Q_{n}\left(M_{r}\right)\right]=0$. Hence, the functions $Q_{n}(x)$ are just the Appell polynomials for the random variable $M_{r}$ in [8]. For Appell functions of any order $\gamma \neq 0$ and related works, see [6] and [12].

(b) For the perpetual American call options, by using a similar approach and Remark 3.1(a), we obtain the optimal stopping boundary $x^{*}$ and the pricing formula in terms of $Q_{g}$ and $f_{M_{r}}$. By a different approach, the solution was obtained earlier by Mordecki [10] for general Lévy processes.

In the following example we consider a special jump-diffusion model so that we can obtain a simple form for the value function.

Example 4.2. Consider the case where $g(x)=\left(x^{+}\right)^{\gamma}$ with $\gamma>1$, and $X_{t}=a t+\sum_{i=1}^{N_{t}^{\lambda}} Y_{i}^{\beta}$, where $a<0$ and $\left\{Y_{i}^{\beta}: i=1,2, \ldots\right\}$ is a sequence of independent exponentially distributed 
random variables with parameter $\beta$. Under these model assumptions, we have $\psi(z)=\mathrm{i} a z-$ $\lambda z /(z+\mathrm{i} \beta)$ and $f_{M_{r}}(y)=d_{0} \delta_{0}(\mathrm{~d} y)+d_{1} \rho_{1} \mathrm{e}^{-\rho_{1} y} \mathrm{~d} y$, where $d_{1}=\left(\beta-\rho_{1}\right) / \beta, d_{0}=\rho_{1} / \beta$, and $\left\{-\mathrm{i} \rho_{1},-\mathrm{i} \tilde{\rho}_{1}\right\}$ are the solutions of $r-\psi(z)=0$. Also, we have

$$
Q_{g}(x)=\frac{-\tilde{\rho}_{1}}{r}\left(-\frac{\lambda \beta}{\beta-\tilde{\rho}_{1}} \int_{x}^{\infty} y^{\gamma} \mathrm{e}^{-\beta(y-x)} \mathrm{d} y-a x^{\gamma}\right)
$$

for every $x>0$. Hence, for each $x<x^{*}$, the value function is given by

$$
V(x)=\frac{-\left(\beta-\rho_{1}\right) \rho_{1} \tilde{\rho}_{1}}{\beta r} \mathrm{e}^{\rho_{1} x} \int_{x^{*}}^{\infty} \mathrm{e}^{-\rho_{1} y}\left(-a y^{\gamma}-\frac{\lambda \beta}{\beta-\tilde{\rho_{1}}} \mathrm{e}^{\beta y} \int_{y}^{\infty} z^{\gamma} \mathrm{e}^{-\beta z} \mathrm{~d} z\right) \mathrm{d} y .
$$

Since

$$
\int_{x^{*}}^{\infty} \mathrm{e}^{\left(\beta-\rho_{1}\right) y} \int_{y}^{\infty} z^{\gamma} \mathrm{e}^{-\beta z} \mathrm{~d} z \mathrm{~d} y=\frac{1}{\beta-\rho_{1}}\left(\int_{x^{*}}^{\infty} \mathrm{e}^{-\rho_{1} z} z^{\gamma}-\mathrm{e}^{\left(\beta-\rho_{1}\right) x^{*}} \int_{x^{*}}^{\infty} \mathrm{e}^{-\beta z} z^{\gamma} \mathrm{d} z\right),
$$

$a=-\lambda \beta /\left(\beta-\rho_{1}\right)\left(\beta-\tilde{\rho}_{1}\right)$, and $Q_{g}\left(x^{*}\right)=0$, we see that

$$
\begin{aligned}
V(x)= & \frac{-\left(\beta-\rho_{1}\right) \rho_{1} \tilde{\rho}_{1}}{\beta r} \mathrm{e}^{\rho_{1} x} \\
\times & {\left[\left(-a-\frac{\lambda \beta}{\left(\beta-\rho_{1}\right)\left(\beta-\tilde{\rho}_{1}\right)}\right) \int_{x^{*}}^{\infty} \mathrm{e}^{-\rho_{1} z} z^{\gamma} \mathrm{d} z\right.} \\
& \left.\left.\quad+\frac{\lambda \beta}{\left(\beta-\rho_{1}\right)\left(\beta-\tilde{\rho}_{1}\right)}\right) \mathrm{e}^{\left(\beta-\rho_{1}\right) x^{*}} \int_{x^{*}}^{\infty} \mathrm{e}^{-\beta z} z^{\gamma} \mathrm{d} z\right] \\
= & \frac{a \tilde{\rho}_{1} \rho_{1}}{\beta r} \mathrm{e}^{\rho_{1}\left(x-x^{*}\right)}\left(x^{*}\right)^{\gamma} .
\end{aligned}
$$

Furthermore, since $\psi(0)=0$ and $r-\psi(z)=(-\mathrm{i} a)\left(z+\mathrm{i} \rho_{1}\right)\left(z+\mathrm{i} \tilde{\rho}_{1}\right) /(z+\mathrm{i} \beta)$, we have $a \tilde{\rho}_{1} \rho_{1} / \beta r=1$ and, hence, $V(x)=\mathrm{e}^{\rho_{1}\left(x-x^{*}\right)}\left(x^{*}\right)^{\gamma}$. Clearly, $V$ is continuous at the optimal boundary $x^{*}$. Since $V^{\prime}\left(x^{*-}\right)=\rho_{1}\left(x^{*}\right)^{\gamma}$ and $g^{\prime}\left(x^{*}\right)=\gamma\left(x^{*}\right)^{\gamma-1}$, there is no smooth fit at $x^{*}$ as $x^{*} \neq \gamma / \rho_{1}$. (To show that $x^{*} \neq \gamma / \rho_{1}$, we set

$$
F(x)=\frac{-\lambda \beta}{a\left(\beta-\tilde{\rho}_{1}\right)} \int_{0}^{\infty}\left(1+\frac{z}{x}\right)^{\gamma} \mathrm{e}^{-\beta z} \mathrm{~d} z .
$$

Then, using the inequality $(1+z / x)^{\gamma} \leq \mathrm{e}^{\gamma z / x}$, we observe that

$$
F(x)<\frac{\lambda \beta}{(-a)\left(\beta-\tilde{\rho}_{1}\right)} \frac{1}{\beta-\gamma / x} .
$$

This implies that $F\left(\gamma / \rho_{1}\right)<\lambda \beta /(-a)\left(\beta-\tilde{\rho}_{1}\right)\left(\beta-\rho_{1}\right)=1$ and, hence, $Q_{g}\left(\gamma / \rho_{1}\right)>0$. Consequently, $x^{*}<\gamma / \rho_{1}$. ) Note that $\{0\}$ is not regular for the half-line $(0, \infty)$ for the process $\left\{X_{t}\right\}$. Our results show no contradiction with the general results of Theorem 5.1 of [13]. Similar results were obtained for the $r=0$ case by Moddecki and Salminen [11].

Example 4.3. Consider the optimal stopping problem (1.1) with $g(x)=\ln (x+1) \mathbf{1}_{\{x \geq 0\}}$. To check conditions (a)-(b) of Theorem 3.2, we first substitute $g(x)=\ln (x+1) \mathbf{1}_{\{x \geq 0\}}$ into (3.17). Multiplying both sides of (3.17) by $(\ln (x+1))^{-1}$ and using Remark 3.1(a), we see that 
$\lim _{x \rightarrow \infty} Q_{g}(x)(\ln (x+1))^{-1}=\left(\psi_{r}^{+}(0)\right)^{-1}=1$, which implies that $\lim _{x \rightarrow \infty} Q_{g}(x)=\infty$. Next, observe that, for $x>0$ and $u>0$,

$$
\left(\frac{g(u+x)}{g(x)}\right)^{\prime}=\frac{\ln (x+1) /(u+x+1)-\ln (u+x+1) /(x+1)}{(\ln (x+1))^{2}}<0
$$

and

$$
\left(\frac{g^{\prime}(x)}{g(x)}\right)^{\prime}=\frac{-(x+1)^{-2} \ln (x+1)-(x+1)^{-2}}{(\ln (x+1))^{-2}}<0 .
$$

By Theorem 3.2, there exists a unique $x^{*}>0$ such that $Q_{g}\left(x^{*}\right)=0$ and $\tau^{*}:=\inf \{t \geq$ $\left.0: X_{t} \geq x^{*}\right\}$ is the optimal stopping time for the optimal stopping problem (1.1) with $g(x)=$ $\ln (x+1) \mathbf{1}_{\{x \geq 0\}}$.

\section{References}

[1] Alili, L. And Kyprianou, A. E. (2005). Some remarks on first passage of Lévy processes, the American put and pasting principles. Ann. Appl. Prob. 15, 2062-2080.

[2] Asmussen, S. And Albrecher, H. (2010). Ruin Probabilities, 2nd edn. World Scientific, Hackensack, NJ.

[3] Asmussen, S., Avram, F. And Pistorius, M. R. (2004). Russian and American put options under exponential phase-type Lévy models. Stoch. Process. Appl. 109, 79-111.

[4] Boyarchenko, S. I. And LeVendorskiǏ, S. Z. (2005). American options: the EPV pricing model. Ann. Finance 1, 267-292.

[5] Chen, Y.-T. AND Sheu, Y.-C. (2009). A note on $r$-balayages of matrix-exponential Lévy processes. Electron. Commun. Prob. 14, 165-175.

[6] Deligiannidis, G., Le, H. And Utev, S. (2009). Optimal stopping for processes with independent increments, and applications. J. Appl. Prob. 46, 1130-1145.

[7] IVAnovs, J. (2011). One-sided Markov additive processes and related exit problems. Doctoral Thesis, University of Amsterdam.

[8] Kyprianou, A. E. And Surya, B. A. (2005). On the Novikov-Shiryaev optimal stopping problems in continuous time. Electron. Commun. Prob. 10, 146-154.

[9] LEWIS, A. L. AND MoRdecKI, E. (2008). Wiener-Hopf factorization for Lévy processes having positive jumps with rational transforms. J. Appl. Prob. 45, 118-134.

[10] MordecKi, E. (2002). Optimal stopping and perpetual options for Lévy processes. Finance Stoch. 6, $473-493$.

[11] Mordecki, E. and Salminen, P. (2007). Optimal stopping of Hunt and Lévy processes. Stochastics 79, $233-251$.

[12] Novikov, A and Shiryaev, A. (2007). On a solution of the optimal stopping problem for processes with independent increments. Stochastics 79, 393-406.

[13] Surya, B. A. (2007). An approach for solving perpetual optimal stopping problems driven by Lévy processes. Stochastics 79, 337-361. 